\title{
Understanding the role of convenience in consumer food choices: a review article
}

\author{
Mary-Ellen Wales
}

\begin{abstract}
The following manuscript was prepared for AGEC 4360, under the supervision of Andreas Boecker, Assistant Professor,
\end{abstract} Department of Food, Agricultural and Resource Economics, Ontario Agricultural College.

$I_{\mathrm{t}}^{\mathrm{t}}$ is evident that convenience plays a prominent role in the food choices of today's consumers. A trend having begun throughout the Western world, consumer demand for convenience foods is now on the rise around the globe. The growing presence of drive-thru windows, microwave dinners, take-out meals, home delivery for groceries and internet shopping, all demonstrate the importance of convenience in determining food choices. ${ }^{8,9}$ Costa et al. have argued that convenience itself determines where, when, why, what, how, and even with whom we eat. ${ }^{5}$ Several studies have examined the role that convenience plays in determining food choices, in particular, studies looking at the role of convenience in relation to full meal preparation and/ or consumption. ${ }^{3,5,6} \mathrm{As}$ an example, Jaeger and Mieselman investigated the construct of food-related convenience itself, looking over each stage of the meal preparation process, and discovered that perceptions of convenience are related to both time and effort. ${ }^{9}$

Convenience has an immense impact on the food choices of today's consumers. This suggests that food products offering less convenience will be deemed less preferable to consumers. Therefore, adding convenience traits to certain products deemed healthy and/or beneficial, such as fresh fruits and vegetables $(\mathrm{FF} \& \mathrm{~V})$, could increase the consumption of these specific food products. It has been argued for many individuals that convenience is a barrier to achieving proper nutrition, using adequate servings of fruits and vegetables as an example. ${ }^{8}$ This relationship, existing between adequate health and fruit and vegetable consumption has been clearly documented, 4, 17, 18, 19 and both the World Health Organization and Food and Agriculture Organization of the United Nations recommend $400 \mathrm{~g}$ of fruits and vegetables per day to prevent chronic diseases such as heart disease, cancer, diabetes, in addition to numerous micronutrient deficiencies. ${ }^{21}$ In 2007, Canada's revised Food Guide was released with fruit and vegetables replacing grains as the primary dietary food group, recommending 7-8 and 810 servings of fruit and vegetables for females and males aged 19-50 years, respectively.

Despite the many health claims of a diet rich in fruits and vegetables, combined with both national and international diet recommendations, the Public Health Agency of Canada reports that roughly 60 percent of Canadians consume low levels of FF\&V. ${ }^{14}$ Hence, this research will investigate the role of convenience in determining FF\&V purchasing and/or consumption. As convenience plays an important role in food choice behaviours, understanding its role in relation to FF\&V could lead to an increased consumption of these products, and consequently, better health for Canadians of all ages.

The original concept of this paper was to focus on awayfrom-home-consumption of all types of food products, however, for two reasons, it was decided to focus solely on FF\&V as a particular food category. First, there is consensus that current fruit and vegetable consumption levels among Canadians are too low, thus leading to poor health and - in combination with an over-consumption of less healthy foods - an increase in diet-related diseases. Second, while there is some literature addressing the factors relating to individuals and their insufficient consumption levels, convenience in relation to $\mathrm{FF} \& \mathrm{~V}$ has received little attention. It is thus necessary to get a broader understanding of the factors involved in the decision process of the consumers. The aims of this paper are twofold. It is evident that convenience in relation to food purchasing and consumption is a complex construct composing of many different dimensions. Thus, this paper will aim to provide a deeper understanding of convenience during the meal preparation and consumption processes, while giving particular attention to $\mathrm{FF} \& \mathrm{~V}$ as a food category. The two areas of investigation will be 1) the dimensions of convenience as a part of the meal preparation and consumption processe, and 2) the individual characteristics of consumers and how they value these dimensions.

\section{DIMENSIONS OF CONVENIENCE}

\section{Time and effort}

Existing research illustrates that there are many dimensions of food related convenience. ${ }^{3,6,8,16}$ Although a consensus on the exact meaning of convenience has yet to be reached, 
many have agreed that convenience includes dimensions of both time and effort saving. 2, 3, 8, 16 As Candel states, transferring culinary skills from the home kitchen to the food distributor or processor can be viewed as contributing to both time and effort saving during the food delivery process. ${ }^{3}$ Jaeger and Mieselman, who conducted a study investigating the perceptions of females towards nine different evening meal scenarios, demonstrated that both time (e.g. long cooking, long preparation) and effort (e.g. work, planning) contributed to perceived inconvenience. ${ }^{9}$ The researchers also went on to describe how perceived time and effort were extremely interdependent across the nine evening meal scenarios. ${ }^{9}$

Effort was further characterized to include both mental and physical effort, being composed of both thinking activities, and the amount of physical energy involved in the meal delivery process. ${ }^{3}$ Table 1 provides examples of the differences between time and effort in regards to meal preparation.

Jaeger and Cardello argue that a 'meal perspective' should be adopted when analyzing food-related convenience, rather than the 'product perspective' used in the majority of literature on the issue. ${ }^{8}$ This allows taking the dimension of 'timing of convenience' into account, as convenience is experienced during one or more stages of the meal preparation and consumption processes. ${ }^{\text {a }}$ This has been contended so that meal characteristics, rather than product characteristics, influence perceived convenience.

Consequently, Jaeger and Cardello see time, effort, and perceived (in)convenience as separate constructs, such that the convenience attributes are not only perceived and measured in single stages, but rather, are spread over the food preparation and consumption processes. ${ }^{8}$ Therefore, the decision to purchase the product is embedded in the broader meal situation when a food product is purchased.

\section{Stages of preparation and consumption process}

An additional dimension of food convenience has also been established, and refers to the different stages of the home food production chain. ${ }^{\text {b, } 3,8,16}$ The five recognized stages of the home food production chain have been recorded in Table 1, and include: planning, purchasing, preparation/cooking, eating and disposal/tidy-up. Additionally, the storing and handling phase can be considered a separate production stage, as these refer specifically to the time, physical, and mental effort required to store and handle food items. Using various examples of time, physical, and mental effort saved during the separate food delivery stages, Table 1 displays the two main convenience dimensions.

To illustrate an example: a consumer decides that she would like to have a vegetable lasagne for an evening meal. During the planning stage of the food production chain, a list is made containing the ingredients needed to make the lasagne, which in turn saves her time and physical effort spent while at the grocery store, as she knows exactly what is needed and spends less time and energy walking through the store. During the purchasing stage, she decides to use the self-checkout, saving her both time and physical effort, as it is faster than the cashier line. When at home, physical and mental effort is saved during the storing and handling stage, as she has purchased vegetables in suitable storing containers, and therefore does not have to take the time to think about how to store the produce. During the preparation stage, mental effort is saved as she has opted to purchase noodles which have detailed cooking instructions on the package, while time and physical effort are saved because she has also opted to purchase pre-cut and pre-washed vegetables. During the eating stage, mental effort is saved as her children are familiar with the lasagne and thus contently eat it. Lastly, during the disposal/ tidy-up stage, time and physical effort are saved through the use of a dishwasher to clean the utensils and dishes used during the eating stage.

\section{Location of convenience production in supply chain or proximity to consumer}

The third notable dimension of food related convenience involves the production aspect of meal related convenience. As Scholderer states, the food producer, the retailer, or food service provider, are different actors of the food chain, that can be responsible for the added convenience of food products. ${ }^{16}$ As an example of added convenience, a peach farm labourer (food producer) selecting peaches based on uniform size and quality, placing them in a basket for easier handling and storage purposes, later saves a consumer time and physical effort at the purchasing stage of the food chain. An employee at a retail grocery store, cutting, washing, and placing some of the peaches in a plastic bowl for immediate consumption, would be an example of added convenience from the retail stage of the food chain. Candel found that consumers oriented towards convenience are more apt to forgo certain food preparation activities altogether by "transferring these to the food processor" thus showing the importance of added convenience from the production and retail sectors of meal production. ${ }^{3}$ Furthermore, an additional actor within the food chain, the food service provider, will add convenience to food products in a number of ways. First of all, there are many different types of food service providers, including, but not limited to: fast food restaurants, true restaurants, cafés, and pubs, which offer numerous types of convenience added products and/ or meals. Examples of the products and/ or meals that could be offered are: a sit down meal, take away food, and coffee to go. In their study of Irish convenience food consumer and four different convenience food categories, De Boer et al. found that restaurant and pub meals were based more on socializing, while ready-made meals were found to be linked most with convenience. ${ }^{6}$ 
Table 1: Convenience in meal preparation, in regards to fruit and vegetable consumption

\begin{tabular}{|c|c|c|c|}
\hline \multirow{2}{*}{$\begin{array}{l}\text { Consumption/ Meal } \\
\text { Production Stage }\end{array}$} & \multicolumn{3}{|c|}{ What is being saved? } \\
\hline & Time & Physical Effort & Mental Effort \\
\hline Planning & $\begin{array}{l}\text { Food item lists, } \\
\text { meal plans }\end{array}$ & Fewer purchasing trips & $\begin{array}{l}\text { Room for spontaneous decisions, no } \\
\text { complex planning }\end{array}$ \\
\hline Purchasing & $\begin{array}{l}\text { Larger retailers with more } \\
\text { items under one roof, } \\
\text { internet shopping, self } \\
\text { check-out facilities }\end{array}$ & $\begin{array}{l}\text { Internet shopping usually } \\
\text { home-delivery based, } \\
\text { shopping carts allow for the } \\
\text { transportation/purchase of } \\
\text { many items at one time }\end{array}$ & $\begin{array}{l}\text { Knowing where to find products } \\
\text { when shopping, familiar packaging }\end{array}$ \\
\hline Storing/ Handling & $\begin{array}{l}\text { No education needed about } \\
\text { how to store fresh produce } \\
\text { properly, no preserves to be } \\
\text { made at home, no monitoring } \\
\text { of stored items needed }\end{array}$ & $\begin{array}{l}\text { Less weight to be handled } \\
\text { (e.g. skins and non-digestible } \\
\text { part of product), fewer/ no } \\
\text { throwing out of expired } \\
\text { produce or staples }\end{array}$ & $\begin{array}{l}\text { Less deterrence caused by spoiled } \\
\text { produce or staples, }\end{array}$ \\
\hline Preparation (Cooking) & $\begin{array}{l}\text { Pre-cut and washed fruits } \\
\text { and vegetables, } \\
\text { kitchen tools such as food } \\
\text { processors, microwaves, etc. } \\
\text { speed up preparation } \\
\text { activities, Take out, pre- } \\
\text { made meals, ready bake food } \\
\text { items, microwave dishes, } \\
\text { marinated items, dishes with } \\
\text { shorter cooking times }\end{array}$ & $\begin{array}{l}\text { Physical effort saved when } \\
\text { preparation activities such as } \\
\text { chopping, peeling, etc. } \\
\text { completed by food service } \\
\text { provider, } \\
\text { less physical effort required } \\
\text { through the use of kitchen } \\
\text { tools }\end{array}$ & $\begin{array}{l}\text { Less knowledge regarding food } \\
\text { preparation required when prepared } \\
\text { items purchased, recipes/ cooking } \\
\text { instructions on food packaging, safe- } \\
\text { handling instructions }\end{array}$ \\
\hline Eating & $\begin{array}{l}\text { Containers suitable for } \\
\text { eating, }\end{array}$ & $\begin{array}{l}\text { Bite-sized food, deboned } \\
\text { meat, skinless/ boneless meat }\end{array}$ & $\begin{array}{l}\text { Familiar food that children like or } \\
\text { can be replaced quickly if they don't } \\
\text { like it }\end{array}$ \\
\hline Disposal/ Tidy-up & $\begin{array}{l}\text { Throw away containers and } \\
\text { utensils, } \\
\text { Items purchased with proper } \\
\text { storage container }\end{array}$ & Dishwashers & $\begin{array}{l}\text { Fewer family disputes regarding tidy- } \\
\text { up duties }\end{array}$ \\
\hline
\end{tabular}

When discussing the location of added convenience, it is important to mention that in some cases, trust in particular players of the food supply chain may be vital to consumers, ultimately affecting their purchasing decisions. For instance, added convenience at the retail level (e.g. grocery stores) would make consumers aware of where exactly the convenience attributes were added, allowing them to make judgements on the cleanliness and the potential food safety measures of the establishment. Thus, in the case of FF\&V purchasing, the location of added convenience may play a significant role in determining consumers' purchasing decisions. Similarly, location of added convenience may also be deemed important in situations where consumers have preferences for particular stages of added convenience. For example, a consumer may prefer purchasing items where convenience attributes have been added by the primary producers themselves, rather than other players of the food chain, e.g. as documented by a "fair trade" label.

\section{Beneficiary of convenience}

A further dimension relating to convenience-orientation can be applied to the person(s) whose effort and time are being reduced. As Scholderer \& Grunert state, the different stages of meal production (planning, purchasing, preparation, cooking, eating, and disposal) can be carried out by different members of the family, which implies that the various stages of effort and/or time reduction can be subsequently reduced by different persons. ${ }^{16}$ Thus, it is important to note that the person(s) who completes meal production activities with reduced time and/or effort depends on the stage of meal production for which they are responsible. As an example, to reduce time and effort, the primary household shopper, choosing to shop at a larger retailer instead of several smaller shops, would imply an element of added convenience solely for themselves. Also, if the primary shopper and their partner are usually involved in the preparation stage of meal production, using a kitchen tool such as a blender could mean that both members could have reduced time and/or physical effort. If the couple and their children eat a meal together containing familiar foods, it is plausible that all members of the family would have reduced mental effort, and thus added convenience, if they are fond of the food itself.

Another example of reduced mental effort during meal production has been stated by Wilk, where, in response to a claim made by Sidney Mintz, he has argued that "various forms of convenience foods have the potential to solve social problems at the dinner table and prevent food fighting." He 
Table 2: factors found to contribute to convenience orientation

\begin{tabular}{|l|}
\hline Socio-economic and demographic \\
\hline $\begin{array}{l}\text { Working status } \\
\text { (Economic) resources } \\
\text { Household size } \\
\text { Presence of children } \\
\text { Food preparation skills and knowledge }\end{array}$ \\
\hline Psychographic \\
\hline $\begin{array}{l}\text { Enjoyment of meal preparation } \\
\text { Involvement (in meal preparation activities) }\end{array}$ \\
\hline
\end{tabular}

further stated that people living in the developed world know that the convenience food items they are eating are generally unhealthy and without taste. But for many people, that is the trade-off for added convenience.

\section{Situational Influence of Meal Situations}

Recent studies have also investigated the situational influence of meal situations as another dimension of food related convenience. ${ }^{6,20}$ The situational influence of eating situations can include the social surroundings, the timing, and the type of situation (snack, full meal and in-between consumption). Verlegh \& Candel found that 'social situations' impacted the intention of consumers' consumption of convenience foods (TV dinners), reporting that consumption intention decreased from dinners eaten alone, with family, and with friends, whereas contrastingly, the intention to consume TV dinners did not differ between 'time-related' situations (weekdays or weekends). ${ }^{20}$ The researchers also found that the context in which TV dinners were consumed (whether alone, with family, or with friends) had an impact on the attributes of the dinner. Similarly, through their research looking at Irish consumers and four different consumer food categories, De Boer et al. found that restaurant and pub meals were less associated with convenience dimensions and more with social events. ${ }^{6}$ Ready and take-away meals, on the other hand, were most associated with convenience related dimensions.

\section{INDIVIDUAL DETERMINANTS OF CONVENIENCE DEMAND}

Research has shown that there are many factors at the individual level contributing to the increased demand of convenience food items. $3,6,7,9,20$ As Candel states, "convenience orientation is an individual characteristic in which consumers can differ." In a study conducted by M. De Boer et al., the researchers divided respondents into groups based on their level of convenience product consumption, ${ }^{6}$ and the results confirmed that the consumers' purchasing of ready meals depended on their lifestyles and beliefs on the purchase on convenience foods. Their analysis was able to demonstrate the extent to which individuals can differ in relation to their purchase of convenience food products. Similarly, Ryan et al. revealed differences towards convenience orientation between six established consumer segments in the Irish population. ${ }^{15}$ The established groups included: hedonistic food consumers (28\%), conservative food consumers (21\%), extremely uninvolved food consumers $(16 \%)$, enthusiastic food consumers $(14 \%)$, moderate food consumers (13\%), and adventurous food consumers $(8 \%)$. It was found that the hedonistic, extremely uninvolved and adventurous food consumers were most convenience oriented out of the six groups, displaying that differences were present between the groups' convenienceorientation.

As has been demonstrated, consumers can be divided successfully into groups based on their purchasing behaviours and attitudes towards food, supporting the idea that convenience orientation differs from person to person. Research to date has divided the factors affecting consumers' convenience orientation into two groups, namely, 'sociodemographic' and 'psychographic' (Table 2).

In addition, existing research has explored how perceived resources can affect convenience orientation, $3,8,20$ and it was found that perceived (in)convenience and resource constraints can contribute to convenience behaviour of meal delivery. Jaeger and Cardello examined the concept of food convenience, specifically within the institutional framework of military feeding in the United States, and presented respondents with meal scenarios reflecting field food provisioning practises. $^{8}$ Jaeger and Cardello found that perceived (in)convenience, time, and effort were separate constructs, which illustrated the fact that perceived (in)convenience of a consumption situation occurs across one or more stages in the process, and that the situational characteristics of a meal also affect perceived (in) convenience. ${ }^{8}$

Scholderer and Grunert developed a model that measured the influence that perceptions of resource constraints have on convenience behaviour among both French $(n=1000)$ and UK $(n=1000)$ respondents. ${ }^{20}$ The researchers concluded that objective household resources had an indirect effect on convenience shopping and product usage, and that this effect was mediated by the perceived objective household resources of respondents, which in turn affected convenience behaviours. ${ }^{20}$ When looking at monetary resources, it was noted that disposable income, employment status, and family size all had an effect on perceived monetary resources. It was also found that family composition (number of adults and children in the household) played the most significant role in resource perception. The researchers state that 
"the persistence of these effects, even when employment status and income are controlled for, suggests that the demands of family life as such may operate in an amplifying way on consumer's perceptions, make family life appear subjectively as an even larger drain on household resources than suggested by subjective data alone." 20

Thus, the research put forth demonstrates the effect that perceived resources have on convenience behaviour, rather than objective resources.

Similarly, Candel's concept of role overload is in line with the above findings. ${ }^{3}$ Role overload is defined as "the degree to which the amount of energy and time demanded of a person as a result of the roles this person performs is perceived to exceed his or her available time and energy resources" and portrays how social structures (such as family) can influence individuals' behaviour. Essentially, the more a person perceives time and energy resources as lacking (due to demands or social pressures) the more a person experiences role overload. The component of role overload linked to internal causes, was found to have a positive correlation with convenience orientation $(\mathrm{P}<0.001)$ suggesting that respondents who experienced role overload, due to their own shortcomings, were more apt to save time and energy during meal preparation activities. Further, the correlation between role overload and external causes was not significant $(\mathrm{P}>0.05)$. Candel distinguishes external causes from internal ones, as external causes are those which a respondent feels they don't have control over, such as others requiring too much of a person's time. ${ }^{3}$ Conversely, not being able to meet everyone's expectations, or having to overextend oneself, are examples of internal causes.

It must also be pointed out that cultural factors also play an active role in determining individuals' convenience orientation. Different cultures have different attitudes and behaviours towards food preparation and consumption. Consequently, it can be argued that attitudes and behaviours towards convenience orientation will also differ between cultures. Recent studies have explored this occurrence, for example, Olsen et al. investigated cultural differences between five European countries in the meaning of convenience orientation and the relationship between convenience, attitudes, and fish consumption. ${ }^{13}$ It was found that actual levels of convenience orientation differed from country to country. However, the researchers pointed out that the meaning of convenience was not culture specific. These findings suggest that the meaning of convenience is steadfast, while the extent to which a person is convenienceoriented can depend on the culture he or she is a part of.

A conceptual model can be developed based on the above discussion regarding convenience orientation.
Convenience orientation is a multidimensional construct in which socio-demographic, psychographic, and perceived resources are factors contributing to its conceptualization.

\section{SOCIO-ECONOMIC AND DEMOGRAPHIC FACTORS}

\section{Working Status}

Working status has been shown to be a major determinant of individual convenience orientation, $3,16,20$ and it was found that the use of convenience appliances and meals eaten away from home was higher if the person responsible for meal preparation held a job. ${ }^{20}$ Verlegh and Candel also found that there was a positive correlation between number of hours worked and consumption frequency, and that consumption of TV dinners was in fact higher if the respondent was employed. ${ }^{20}$ Candel discovered that only meal preparers who work over 30 hours per week were more significantly convenience-oriented than those who worked less than 9 hours per week. ${ }^{3}$ Respondents who worked less than 9 hours per week scored the lowest on the convenience-orientation scale; even lower than those who were unemployed. Perhaps, respondents working fewer than 9 hours per week chose the job simply to add an interesting element to their lives, explaining why they were found to score lower on the convenience-orientation scale than their unemployed counterparts. ${ }^{3}$ This is in line with Scholderer and Grunert, who also discovered that unemployment did not necessarily increase a person's perceived time budget. $^{20}$

\section{Resources}

Household income is considered a major determinant of convenience orientation, with higher incomes possessing higher convenience orientation and consumption. ${ }^{1,2,20}$ When discussing the Irish food market and changing eating patterns in Ireland, Ryan et al. describes how people with large disposable incomes, and very little time to spend, are often categorized as the "cash rich, time poor consumers." 15 This description is certainly applicable to the rest of the Western world. When speaking of variances between the purchasing of convenience items, and food related lifestyle segments, the researchers also stated that the differences in categories were due to the larger proportion of consumers with higher income levels in those groups, which purchased more convenience items. $^{15}$

Women's participation in the labour market is another determinant, especially when more than thirty hours a week are worked. As Ryan et al. states, the rise in the number of women working outside the home has had a significant effect on traditional food consumption patterns. ${ }^{15}$ Not only are dual income families busy, they have a greater income to spend on convenience food items. 


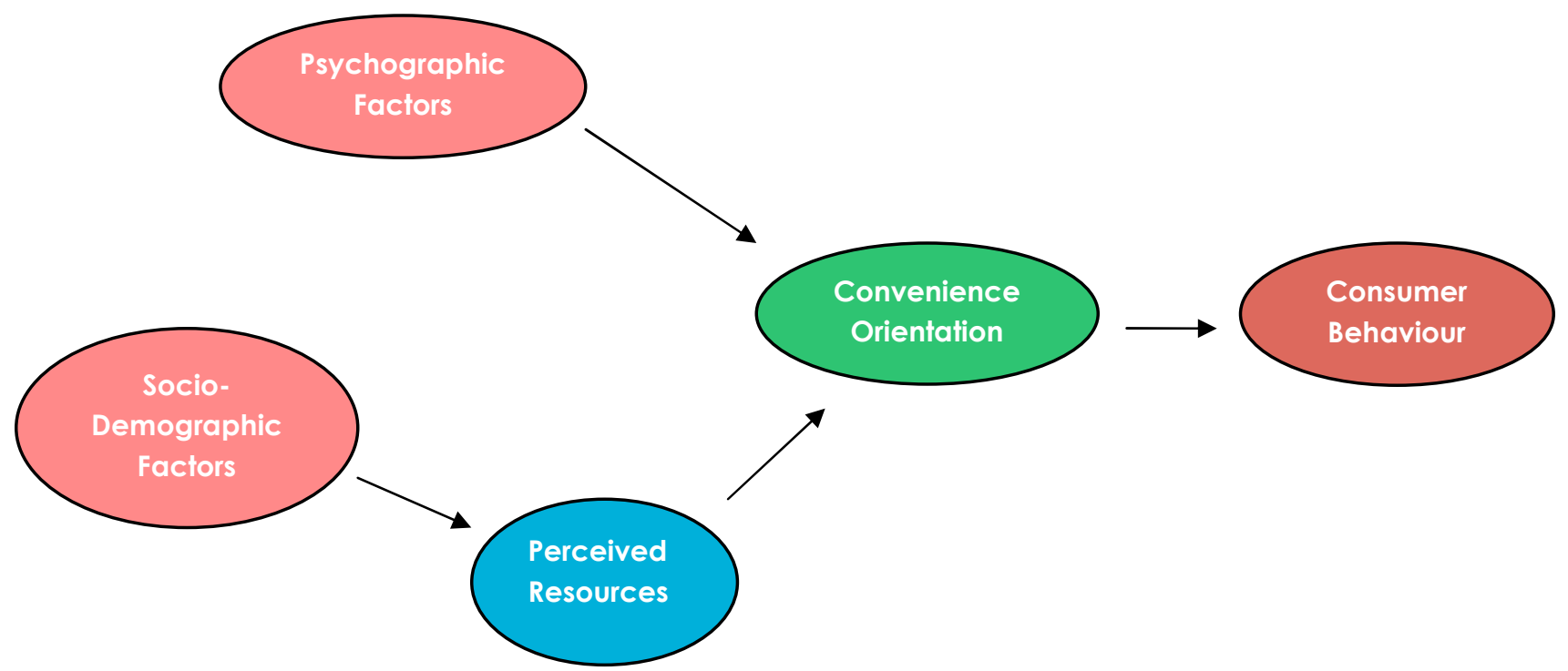

Figure 1: Convenience Orientation Model. Adapted from Scholderer and Grunert (2005: 109).

\section{Household size}

Another factor affecting convenience orientation is that of household size. Candel found that singles were more convenience-oriented than multiple-person households, while there was no significant difference between multiple-person households of various sizes. ${ }^{3}$ He states that those respondents who were not responsible for looking after other household members in regards to meal preparation, but were responsible for both earning a living and housekeeping, were most convenience oriented. A study completed by Marquis confirmed the importance of convenience in determining food choice among young, single adults living in residence halls. It was found that convenience was the most important food choice factor, while price, pleasure, health, and concern about weight followed after. ${ }^{11}$ Similarly, Marquis and Manceau determined that convenience played a big role in determining food choices of single men living in apartments in Montreal. ${ }^{12}$

\section{Presence of children}

In relation to household size, the presence of children also seems to play a role in determining convenience orientation. Candel discovered that the presence of children had an effect on convenience orientation, as meal preparers with children were found to be less convenience oriented. ${ }^{3}$ This is somewhat surprising as it could be assumed that households with children are more time constrained, and thus perhaps more convenience-oriented than families without children. However, the opposite has been found, as families with children appear to be less convenience oriented than those without. Perhaps families with children are more inclined to want to perceive meal preparation and cooking as an enjoyable family activity when children are involved in the process. It could also be that individuals generally feel that it is more worthwhile to cook for one or two other people rather than solely for themselves.

\section{Food Preparation Skills and Knowledge}

A cross sectional analysis of males $(n=764)$ and females $(n=964)$ aged 18-23 completed by Larson et al. discovered that young adults reporting frequent food preparation skills displayed less frequent fast food consumption. ${ }^{10}$ The results of this study suggest that food preparation skills can have an impact on convenience food consumption, as fast food is a commonly consumed convenience food. The researchers also found that respondents reporting frequent food preparation skills were also more likely to meet the dietary requirements for fat, fruits, vegetables, calcium and whole grains of the Healthy People 2010. ${ }^{c, 10}$ Differences between food preparation skill levels and fruit and vegetable intake were found to be most apparent. $31 \%$ of respondents who reported high levels of food preparation skills were consuming five servings of fruits and vegetable servings per day, in comparison with only $3 \%$ who reported the lowest level of cooking skills. In conclusion, the researchers support and recommend programs and interventions aimed at improving food preparation skills as a way to improve dietary intake. ${ }^{10}$

\section{PSYCHOGRAPHIC FACTORS}

\section{Enjoyment of Meal Preparation}

Candel measured the effect that enjoyment of meal preparation can have on a person's convenience orientation. ${ }^{3}$ He defined enjoyment of meal preparation as the extent to which a person receives pleasure from meal preparation activities, and it was found that enjoyment of meal 
preparation was negatively related to convenience orientation. ${ }^{3}$ Similarly, Buckley et al. divided UK consumers into four segments based on their 'consumer food lifestyles' (CFL): the food connoisseurs (26\%), the home meal preparers $(25 \%)$, the kitchen evaders $(16 \%)$, and the convenience seeking grazers $(33 \%) .{ }^{2}$ To illustrate, the 'food connoisseurs' were characterized as deriving the most pleasure from experimentation during meal preparation and new foods. It was found that respondents within this group were less likely than those in the remaining groups to distinguish convenience foods as vital or healthy. The group was also the least inclined to feel that convenience food are time saving in comparison to the other groups. Thus, it is evident from existing literature that enjoyment of meal preparation has a negative impact on consumers' convenience orientation of food products.

\section{Involvement}

Involvement is another individual determinant argued to have an effect on convenience orientation. Candel refers to involvement as the "degree to which an individual finds food products important" which has been found to have a negative effect on convenience orientation. ${ }^{3}$ Ryan et al. concluded similar results, where respondents were divided into six consumer segments based on their purchases, preparation and consumption of food products in general. ${ }^{15}$ It was found that the 'extremely uninvolved' segment purchased convenient food products, such as frozen vegetables, prepared salad, canned food and prepared food, more often than any other consumer segment. This group was characterized as uninterested in shopping and cooking, and possessed a negative attitude towards eating out in general. Whether or not an individual finds food products important therefore impacts individual convenience orientation.

On the whole, attitudes and values towards meal preparation have an impact on individuals' convenience orientation. Existing literature has been able to group consumers into segments based on their lifestyles and attitudes towards food and convenience foods, as well as the purchase frequency of these products. ${ }^{2,} 6,15$ Consumers can differ in their perceptions of food and meal preparation, further justifying the fact that convenience-orientation is an individual characteristic in which consumers can differ.

\section{CONClusions AND OUTLOOK ON Future RESEARCH}

Research has shown that convenience in relation to food purchasing and consumption is a complex process composed of many attributes. This paper has focused on two areas with an overall emphasis on fresh fruits and vegetables (FF\&V) as a particular food category: 1) the dimensions of food convenience as part of a meal preparation and consumption process, and, 2) individual characteristics of consumers and how they value these dimensions.

In regards to the dimensions of convenience, convenience has been defined to include aspects of both time and effort saving - with effort consisting of both physical and mental effort. In-depth studies investigating the construct of convenience have generated vocabulary terms centered on both time and effort saving. Thus, in order to increase FF\&V consumption, it is suggested that fruits and vegetables be marketed as both time and effort saving when possible. This could potentially increase consumption, as consumers would perceive FF\&V as time and effort saving much like other prepared foods.

When investigating food related convenience, the adoption of a 'meal perspective', rather than a 'product perspective' has been advocated. This has been proposed since the decision to purchase a food product is part of a broader meal scenario, and therefore, convenience attributes of a product are spread over more than one stage of the food preparation and consumption processes. When focusing on fruits and vegetables as a particular food category, the adoption of a meal perspective is imperative as fruits and, even more so, vegetables are typically part of broader meal scenarios. Thus, when aiming to increase fruit and vegetable purchases, marketers must plan to utilize the meal perspective, and present fruits and vegetables as convenient, both independently and as components of a meal.

More particularly, six stages of the food preparation and consumption process have been recognized and include: planning, purchasing, storing/handling, preparation/cooking, eating and disposal/tidy-up. Table 1 has provided examples of time and effort saved during the six different stages of the food preparation and consumption processes, with an emphasis on fruits and vegetables as a particular food category. As previously stated, a meal perspective should be adopted with regards to increasing fruit and vegetable consumption. The six mentioned stages constitute the meal preparation and consumption processes to which the meal perspective should be applied. When marketing FF\&V, attention should be given to the fact that convenience can spread over the six mentioned stages and therefore, convenience attributes can be applied and advertised to more than one stage of the food preparation and consumption process. Consumers oriented towards convenience are more apt to forgo certain food preparation activities altogether by transferring these to the food processor. Hence, in order to increase consumption, FF\&V can be marketed as saving consumers time and effort over more than one or two of the stages, but rather, over a number of stages.

In regards to individual determinants, this paper has also investigated the individual characteristics of consumers, which have been shown to impact consumers' convenience orientation. The characteristics include two groups: 1) socioeconomic/ demographic (working status, resources, 
household size, presence of children, food preparation skills and knowledge) and 2) psychographic (enjoyment and involvement in meal preparation) factors.

It is clear that resources (i.e. income) and working status do affect convenience orientation; however, the research has also shown the extent to which perceived resources play a role in consumer decision making. With regards to FF\&V consumption, perceived resources may play a role in determining consumer purchasing and consumption behaviour. External strains, such as family and social responsibilities, were found to increase convenience orientation, suggesting that social responsibility may be a significant determinant of convenience-orientation rather than resources and working status alone.

In addition, involvement and enjoyment in meal preparation were shown to affect individuals' convenience orientation. It was found that individuals deriving the most pleasure from meal preparation activities were less likely to perceive convenience foods as time saving, vital or healthy. Involvement in meal preparation was determined to have a negative effect on convenience orientation, as individuals who perceived food products to be important were less inclined to purchase convenience items. In relation to FF\&V consumption, involvement and enjoyment in meal preparation are perhaps traits which may lead to an increased fruit and vegetable consumption, as both have been shown to lead to a decreased consumption in convenience foods. At this stage, more research is needed to subject this conjecture to an appropriate empirical test.

Future research, illustrating the perception and uses of FF\&V, would be useful in determining the stages during the meal and consumption processes to which further convenience traits may be added. This would be beneficial when aiming to increase fruit and vegetable consumption towards current recommended levels, as convenience is a demanded trait of consumers of food products and can thus be applied to the food category of FF\&V. Understanding the perceptions and uses of FF\&V could allow for the specific potential convenience traits to be added to this food category, with more precision and benefit to consumers.

Furthermore, existing research has focused mainly on convenience foods as a food category; however, future researching exploring the relationship between perceived resources and FF\&V consumption alone may point to whether resources alone or, rather, perceived resources, affect FF\&V consumption.

Food preparation and cooking skills have been shown to lead to an increase in fruit and vegetable consumption. It would seem that food preparation knowledge and cooking skills are overlooked as personal assets leading to better health. In regards to FF\&V, food preparation skills and knowledge may be important individual traits leading to increased FF\&V consumption, and thus, greater overall health.

\section{REFERENCES}

1. Berry, L.L., Seiders, K., Grewal, D. (2002): Understanding service convenience. Journal of Marketing, 66(2002): 1-17.

2. Buckley, M., Cowan, C., McCarthy, M. (2007): The convenience food market in Great Britain: Convenience food lifestyle (CFL) segments. Appetite, 49(2007): 600617.

3. Candel, M.J.J.M. (2001): Consumers' convenience orientation towards meal preparation: conceptualization and measurement. Appetite, 36(2001): 15-28.

4. Cohen, J.H., Kristal, A.R., Stanford, J.L. (2000). Fruit and Vegetable Intake and Prostate Cancer Risk. JNCI Journal of the National Cancer Institute, 92(1): 61-68.

5. Costa, A.I. de I., Schoolmeester, D., Dekker, M., Jongen., W.M.F. (2005): To cook or not to cook: A means end study of motives for choice of meal solutions. Food Quality and Preference, 18(2007): 77-88.

6. De Boer, M., McCarthy, M., Cowan, C., Ryan, I. (2003): The individual of lifestyle characteristics and beliefs about convenience food on the demand for convenience foods in the Irish market. Food Quality and Preference, 15(2004): 155-165.

7. Jabs, J., Devine, C.M. (2006): Time scarcity and food choices: An overview. Appetite, 47(2006): 196-204.

8. Jaeger, S.R., Cardello, A., V. (2007): A construct analysis of meal convenience applied to military foods. Appetite 49(1): 231-239.

9. Jaeger, S.R., Meiselman, H., L. (2004): Perceptions of meal convenience: the case of at home evening meals. Appetite, 42(2004): 317-325.

10. Larson, N., Perry, C.L., Story, M., Neumarksztainer, D. (2006): Food Preperation by Young Adults is Associated with better Diet Quality. Journal of the American Dietetic Association, 106: 2001-2007.

11. Marquis, M. (2005): Exploring convenience orientation as a food motivation for college students living in residence halls. International Journal of Consumer Studies, 29(1): 55-63.

12. Marquis, M., Manceau, M. (2007): Individual factors determining the food behaviours of single men living in apartments in Montreal as revealed by photographs and interviews. Journal of Youth Studies, 10(3): 305-316.

13. Olsen, S., V., Scholderer, J., Bruns $\varnothing$, K., Verbeke, W. (2007): Exploring the relationship between convenience and fish consumption: A cross-cultural study. Appetite, 49 (1): 84-91.

14. Public Health Agency of Canada. (2008). Minimizing the Risks of Cardiovascular Disease. [online report], Ottawa, Ontario, Canada (http://www.phacaspc.gc.ca/cd-mc/cvd-mcv/risk-risques-eng.php).

15. Ryan, I., Cowan, C., McCarthy, M., O'Sullivan, C. (2002): Food-Related Lifestyle Segments in Ireland with 
a Convenience Orientation. Journal of International Food \& Agribusiness Marketing, 14(4): 29-47.

16. Scholderer, J., Grunert, K., G. (2004). Consumers, food and convenience: The long way from resrouce constraints to actual consumption patterns. Journal of Economic Psychology, 26 (2005): 105-128.

17. Steinmetz, K.A., Potter, J.D. Vegetables, Fruit and Cancer Prevention: A Review. Journal of the American Dietetic Association, 96(10): 1027-1039.

18. Van Duyn, M. (2000): Overview of the Health Benefits of Fruit and Vegetable Consumption for the Dietetics Professional Selected Literature. Journal of the American Dietetic Association, 100 (12): 1511-1521.

19. van't Veer, P., Jansen, M., Klerk, M., Kok, F. (2000): Fruits and vegetables in the prevention of cancer and cardiovascular disease. Public Health Nutrition, 3(2000): 103-107.

20. Verlegh, P.W.J., Candel, M.J.J.M. (1999): The consumption of convenience foods: reference groups and eating situations. Food Quality and Preference,
10(1999): 457-464.

21. World Health Organization (WHO) \& Food and Agriculture Organization of the United Nations (FAO). (2004): Fruit and vegetables for health: Report of a Joint FAO/WHO Workshop, 1-3 September, Kobe, Japan

(http://www.who.int/dietphysicalactivity/publications/fr uit_vegetables_report.pdf).

\section{ENDNOTES}

a 'Preparation and consumption process' along with the 'home food production chain' will be used interchangeably to refer to the steps involved in the meal process.

${ }^{\mathrm{b}}$ RE: Footnote 2.

c Healthy People 2010 is a national agenda aimed at improving the health of Americans targeting key dietary behaviours (http://www.healthypeople.gov/). 